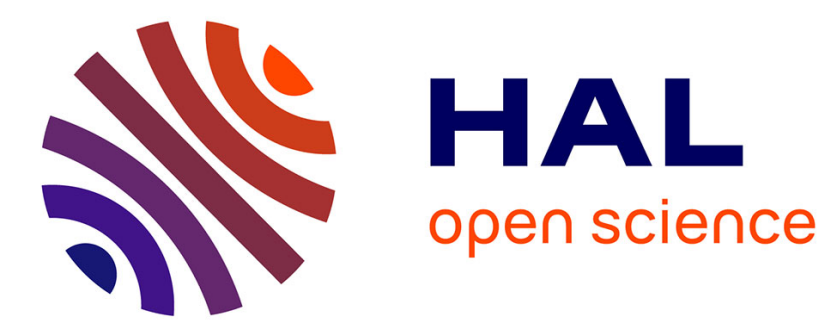

\title{
Lutherie informatique et lutherie acoustique
}

\author{
J. Laplane, P. Guillemain, Richard Kronland-Martinet
}

\section{To cite this version:}

J. Laplane, P. Guillemain, Richard Kronland-Martinet. Lutherie informatique et lutherie acoustique. Journal de Physique IV Proceedings, 1994, 04 (C5), pp.C5-629-C5-632. 10.1051/jp4:19945135 . jpa00252812

\section{HAL Id: jpa-00252812 https://hal.science/jpa-00252812}

Submitted on 1 Jan 1994

HAL is a multi-disciplinary open access archive for the deposit and dissemination of scientific research documents, whether they are published or not. The documents may come from teaching and research institutions in France or abroad, or from public or private research centers.
L'archive ouverte pluridisciplinaire HAL, est destinée au dépôt et à la diffusion de documents scientifiques de niveau recherche, publiés ou non, émanant des établissements d'enseignement et de recherche français ou étrangers, des laboratoires publics ou privés. 


\title{
Lutherie informatique et lutherie acoustique
}

\author{
J. LAPLANE*, P. GUILLEMAIN et R. KRONLAND-MARTINET
}

CNRS-LMA, 31 ch. J. Aiguier, 13402 Marseille cedex 20, France

${ }^{*}$ Atelier de Lutherie, 22 rue de l'Eglise St Michel, 13005 Marseille, France

\begin{abstract}
This paper presents some applications of time-frequency representations to the study of guitar sounds. These techniques allow to estimate acoustically relevant parameters such as frequency and time behaviours of the components. From real guitar sounds recorded in an anechoïc room, we have made a link between the structure modifications of the instrument and sound parameters such as Helmholtz, top-plate and the first harmonic components of the sound. In addition, these techniques allow to re-synthesize independantly each component, giving a new audio-tool to the instrument designer.
\end{abstract}

\section{Introduction}

La réalisation et la mise au point d'un instrument de musique par un luthier fait appel à un ensemble de connaissances acquises au cours d'une longue expérience. Même si l'architecture de la structure sonore et le choix des matériaux répondent à une logique mécanique, ce savoir-faire est le résultat d'une mise en correspondance essentiellement intuitive des propriétés de la structure avec les qualités sonores engendrées par l'instrument. Certaines phases de la mise au point d'un instrument peuvent bénéficier des méthodes modernes d'analyse et de synthèse numérique de signaux sonores.

Ainsi, les techniques d'estimation de lignes spectrales contenues dans un signal à partir de représentations temps-fréquence permettent d'accéder à des grandeurs physiques extrêmement importantes telles la répartition des partiels, les lois de modulation d'amplitude et de fréquence associées à chacune des composantes, la séparation des aspects liés à la source d'excitation (corde vibrante) et à la réponse du système. D'autre part, l'utilisation de méthodes linéaires et isométriques permet d'effectuer des resynthèses partielles obtenues en éliminant certaines contributions spectrales. Ainsi est il possible à partir de l'analyse d'un son réel produit par un instrument de musique d'écouter grâce à la synthèse l'impact psychoacoustique produit par l'adjonction ou la suppression de composantes. De même, on peut, par soustraction des composantes liées au système excitateur, avoir accès à l'aspect percussif engendré par la mise en vibration de la structure lors du jeu instrumental.

Des études visant à fournir au luthier des outils d'analyse-synthèse adaptés à une démarche essentiellement audiophonique ont été menées dans le cas de la guitare. Dans ce cadre, nous allons décrire comment l'utilisation de méthodes d'analyse-synthèse permet d'accéder à une caractérisation fine du son engendré ainsi qu'à sa mise en relation avec les caractéristiques physiques de l'instrument. Nous présenterons les résultats obtenus sur une guitare dans des configurations différentes (attache des cordes, ouverture de la caisse, couplage table-fond par une âme) et montrerons comment l'analyse des sons engendrés permet au luthier d'associer aux modifications de structure les variations sonores correspondantes. En particulier, on s'intéressera au son engendré par les vibrations de la structure, notamment la résonance du premier mode de la table d'harmonie et la résonance de l'air situé dans la caisse [1]. En effet, dès le choix du bois et à chaque étape du façonnage de l'instrument jusqu'à la pose des cordes, le luthier évalue à la percussion du doigt la qualité sonore de la structure qu'il élabore. 


\section{L'analyse temps-fréquence de sons musicaux.}

Le timbre d'un son musical est caractérisé non seulement par la répartition spectrale de ses composantes mais également par leur évolution temporelle. Ainsi, la seule donnée des fréquences et amplitudes moyennes des composantes d'un son ne suffit elle pas à simuler par synthèse ce dernier et n'engendre qu'un son de type "orgue électronique". L'aspect temps-fréquence étant donc inhérent à la description d'un signal musical, les représentations linéaires telles que la transformée en Ondelettes ou la transformée de Gabor jouent un rôle important dans la caractérisation des sons musicaux.

L'idée générale consiste à estimer des spectres locaux (autour d'instants donnés) de façon à obtenir pour chacune des composantes entrant dans la contitution du son deux fonctions temporelles appelées "enveloppes", qui représentent les variations de fréquence et d'amplitude des composantes. Pour cela, on part des représentations désormais classiques telles que Ondelettes ou Gabor qui consistent à décomposer le signal en terme de "grains élémentaires de son" construits de façon à paver l'espace de représentation temps-fréquence. Ainsi pour les ondelettes, les grains $\mathrm{g}_{\mathrm{a}, \tau}(\mathrm{t})$ sont obtenus par dilatation et translation d'une fonction mère $g(t)$ :

$$
g_{a, \tau}(t)=\frac{1}{a} g\left(\frac{t-\tau}{a}\right) \quad a>0
$$

tandis que la transformée de Gabor considère des grains obtenus par translation en temps et en fréquence d'une fonction mère:

$$
\mathrm{g}_{\omega, \tau}(\mathrm{t})=\mathrm{w}(\mathrm{t}-\tau) \exp (\mathrm{i} \omega(\mathrm{t}-\tau)) \quad \text { où } \mathrm{w}(\mathrm{t}) \text { est une fenêtre de localisation temporelle. }
$$

Les représentations temps-fréquence sont alors obtenues par "comparaison" (produit scalaire) du signal et des grains élémentaires:

$$
\begin{aligned}
& S(\tau, a)=\frac{1}{a} \int \bar{g}\left(\frac{t-\tau}{a}\right) s(t) d t \\
& G(\tau, \omega)=\int s(t) \bar{w}(t-\tau) e^{-i \omega}(t-\tau) d t
\end{aligned}
$$

Nous ne discuterons pas ici les propriétés associées à ces différents types de représentation et renvoyons le lecteur aux références [2].

Les représentations obtenues donnent généralement lieu à deux images (le module et la phase de la transformée). Le carré du module s'interprète comme une densité d'énergie répartie dans le plan tempsfréquence, tandis que la phase porte des informations fines sur l'aspect oscillant (fréquence instantanée) des composantes.

La figure 1, représente la transformée de Gabor d'un son de guitare et laisse apparaitre les différentes composantes, tant harmoniques (corde vibrante) qu'inharmoniques (réponse de la structure).

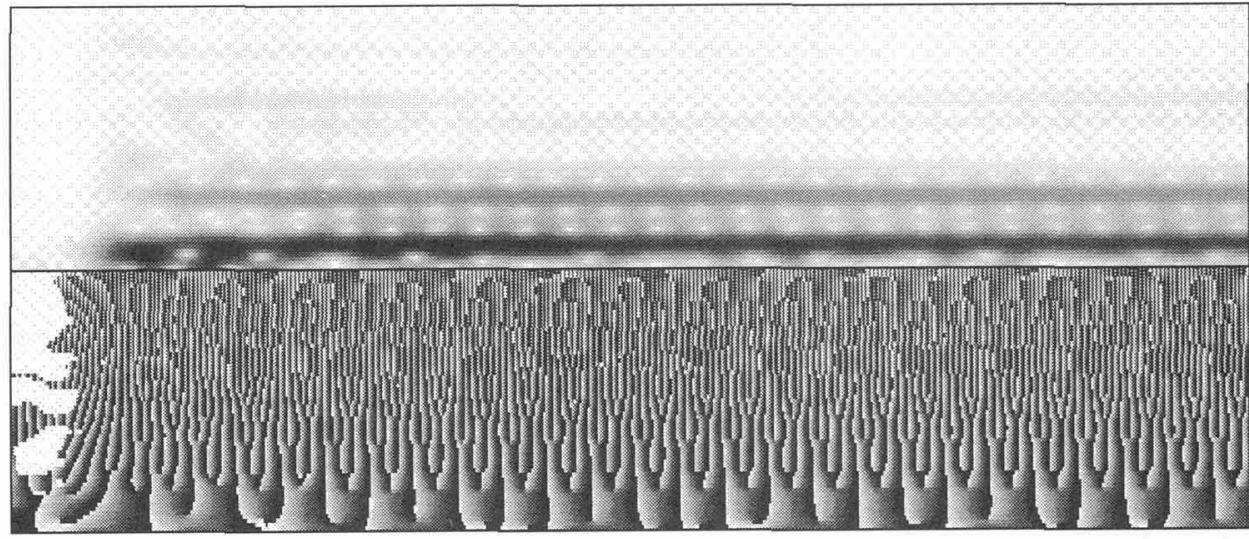

Figure 1: Module et phase d'une transformée de Gabor d'un son de guitare.

De façon à obtenir une information quantitative sur le comportement des composantes, il est possible de construire un banc de filtre adapté à la résolution des composantes individuelles[3]. Pour cela, l'estimation de la fréquence locale de chacune des composantes à partir de la dérivée de la phase de la transformée permet de définir les fréquences centrales et de coupures des filtres estimateurs. Leur 
caractéristique essentielle est de selectionner dans le signal une composante en l'isolant des autres. La sortie de tels filtres permet alors de séparer chaque composante et d'obtenir les enveloppes de fréquence et d'amplitude associées à chacunes d'entre elles. Ces enveloppes sont caractéristiques du timbre sonore et premettent outre une analyse quantitative et comparative des sons, une re-synthèse sélective, permettant de n'écouter par exemple que l'effet transitoire dû à la mise en vibration de la structure.

La figure 2 présente les enveloppes d'amplitudes des quatre premières composantes du son de guitare représenté figure 1 (composante de Helmholtz, premier mode de plaque, premier et second harmonique).
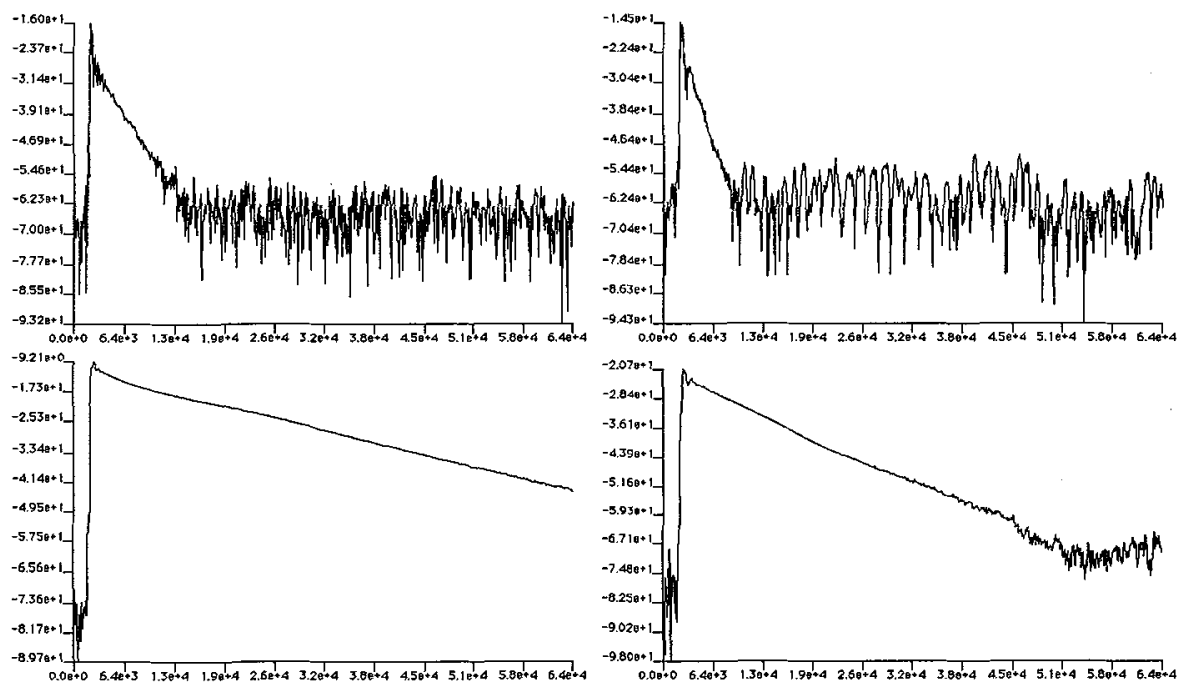

Figure 2: Lois de modulations d'amplitude associées aux composantes de Helmholtz, de plaque et des deux premiers harmoniques de la corde.

\section{Analyse temps-fréquence de sons de guitares.}

Dans cette étude, nous avons cherché à caractériser les variations de timbre apportées par certaines modifications sur la structure d'une guitare. Pour cela, nous avons considéré quatre configurations à partir d'un même instrument. Les différences concernent la présence ou non d'une âme (A) (petit pilier coincé entre la table d'harmonie et le fond), d'une seconde rosace (2R) et l'attache des cordes sur le chevalet (C) ou sur un manche intérieur (MI).

Des mesures ont été effectuées en chambre anéchoïque sur des sons isolés, joués par un guitariste professionnel, afin d'obtenir un mode de jeu aussi constant que possible (point d'impact sur la corde, intensité de l'excitation).

De façon à comparer les résultats, nous présentons ci-dessous, pour chaque configuration:

- les fréquences associées à la résonance de l'air (f-Helmholtz) et au premier mode de résonance de la plaque supérieure (f-plaque),

- les durées de réverbération associées aux modes de Helmholtz (T-Helmholtz) et de plaque ( $\mathrm{T}$ plaque) (durée correspondant à une atténuation de $40 \mathrm{~dB}$ ),

- les durées de réverbération associées aux harmoniques 1 et 2 ( $T$ harm1 et $T$ harm2), de plaque (L1-Lp).

- les niveaux relatifs de l'harmonique 1 par rapport aux niveaux du mode de Helmholtz (L1-Lh) et

De plus, les sons correspondant à MI-2R-A et Mi-2R(1) ont été joués avec une attaque butée tandis que les autres ont été joués avec une attaque pincée.

\begin{tabular}{|c|c|c|c|c|c|c|c|c|}
\hline & f Helmholtz & T Helmholtz & f plaque & T plaque & T harm1 & T harm2 & L1-Lh & L1-Lp \\
\hline C-2R & 97 & 0.39 & 213 & 0.34 & 1.66 & 1.4 & 25.7 & 20.1 \\
\hline MI-1R & 97 & 0.39 & 218 & 0.17 & 1.47 & 1.3 & 26.9 & 27.3 \\
\hline MI-2R-A & 107 & 0.42 & 220 & 0.21 & 2.44 & 1.4 & 23.2 & 15.1 \\
\hline MI-2R(1) & 103 & 0.45 & 218 & 0.17 & 1.53 & 1.5 & 24.5 & 20.3 \\
\hline MI-2R(2) & 103 & 0.33 & 215 & 0.21 & 1.49 & 1.3 & 27.4 & 29.6 \\
\hline
\end{tabular}


Les caractéristiques perçues par le guitariste pour chaque modification de structure peuvent être résumées ainsi:

- la deuxième rosace $2 \mathrm{R}$ (dans l'éclisse supérieure) donne ampleur sonore et détaché des notes en atténuant l'effet de résonance du volume d'air de la caisse. La guitare semble alors se comporter plus comme une caisse d'amplification plutôt qu'une caisse de résonance.

- le manche intérieur MI favorise la puissance, la spontanéité et la durée du son.

- l'âme A semble augmenter la puissance sonore et renforcer le bruit de structure.

Nous allons à présent relier ces modifications de structure et de jeux aux variations des grandeurs mesurées. L'êtude du tableau présenté ci-dessus montre que:

- la seconde rosace $2 \mathrm{R}$ augmente f-Helmholtz, diminue T-Helmholtz et augmente T-plaque. Ceci est dû à une augmentation de la surface ouverte, donc à une fuite d'énergie supplémentaire et à un couplage moindre de la plaque et du volume d'air intérieur (voir MI-1R et MI-2R(2)).

- le manche intérieur MI a pour effet de diminuer T-plaque et d'augmenter L1-Lp (donc diminuer Lp). Ceci est dû à l'inertie du manche intérieur qui est couplé à la plaque par les cordes (voir C-2R et MI$2 \mathrm{R}(2))$.

- la présence de l'âme A provoque une diminution de L1-Lp (donc augmente Lp) et une augmentation de T-plaque. Ceci est dû à une rigidification du haut de la table car l'âme est positionnée sur le noeud du premier mode de résonance de la plaque au milieu de la barre d'harmonie transversale située sous la rosace. L'âme constitue de plus un couplage avec le fond et le contour des éclisses. (voir MI-2R-A et MI2R). L'augmentation de $\mathrm{T} 1$ est particulière à la note jouée car l'harmonique 1 correspond ici à un mode supérieur de résonance de la plaque lorsqu'elle est couplée à l'âme.

- les attaques butées ont pour effet de renforcer les "bruits de structure". Ceci se traduit par une augmentation des niveaux Lh et Lp par rapport au niveau de l'harmonique 1 (H1). De plus, T-plaque diminue (doigt posé sur la corde voisine amortissant la vibration de la plaque).

\section{Conclusion.}

Nous avons présenté ici quelques résultats issus d'une étude qui, bien qu'encore préliminaire, permet de montrer qu'il est possible de mettre en correspondance des effets subjectifs dûs à des modifications de structure avec des paramètres obtenus par des analyses temps-fréquence. Les résultats présentés restent à être généralisés à partir d'une étude systématique sur un grand nombre d'échantillons, ce qui permettrait peut-être d'exhiber des valeurs moyennes des paramètres mesurés correspondant à un son résultant dont l'impact esthétique est optimal.

L'aspect audiophonique procuré par la synthèse est également important car il permet au luthier d'effectuer des écoutes sélectives, privilégiant par exemple les effets transitoires dûs au jeu instrumental par rapport aux effets quasi-périodiques engendrés par la corde vibrante. Une meilleure connaissance de ce qu'apporte des modifications de structure sur l'attaque de la note et son développement harmonique permettra au facteur d'instrument d'adapter sa réalisation à la demande du musicien ou de poursuivre en meilleure connaissance de cause l'esthétique sonore qu'il recherche.

\section{Références}

[1] I.M. Firth "Physics of the guitar at the Helholtz and first top-plate resonances", JASA, V61, n ${ }^{\circ}$, feb 77 , pp 588-593.

[2] "Wavelets and Applications", Meyer ed, RMA series, Masson Springer-Verlag 91.

[3] P. Guillemain, R. Kronland-Martinet. "Additive resynthesis of sounds using continuous timefrequency analysis techniques",Procs. ICMC 10-15 oct 92, San Jose. pp 10-13 